\title{
Effect of interleukin-2 treatment combined with magnetic fluid hyperthermia on Lewis lung cancer-bearing mice
}

\author{
RUNLEI HU ${ }^{1}$, SHENGLIN MA ${ }^{2}$, XIANFU KE ${ }^{3}$, HONG JIANG $^{1}$, DONGSHAN WEI ${ }^{1}$ and WEI WANG ${ }^{1}$ \\ Departments of ${ }^{1}$ Thoracic Surgery and ${ }^{2}$ Radiation Oncology, Hangzhou First People's Hospital, Nanjing Medical University; \\ ${ }^{3}$ Laboratory Animal Center, Zhejiang Academy of Medical Sciences, Hangzhou, Zhejiang 310006, P.R. China
}

Received August 17, 2015; Accepted October 16, 2015

DOI: $10.3892 /$ br. 2015.540

\begin{abstract}
The present study aimed to investigate the therapeutic effect of interleukin-2 (IL-2) treatment combined with magnetic fluid hyperthermia (MFH) on Lewis lung cancer-bearing mice. Magnetic fluids were prepared in vitro and directly injected into the tumors in the mice, which were subjected to an alternating magnetic field. The temperature in the tumor reached $43^{\circ} \mathrm{C}$ and was maintained by controlling the strength of magnetic field for $30 \mathrm{~min}$. Twenty-four hours later, IL-2 was injected directly into the tumors. Mice were divided into four groups: Group I (control), II (MFH), III (IL-2) and IV (IL-2+MFH). The tumor grew gradually in groups II and IV (both $\mathrm{P}<0.05$ ) compared to the control group. Histological analysis showed that the tumor cells underwent apoptosis and necrosis. Immunohistochemistry results demonstrated that heat-shock protein 70 and cluster of differentiation (CD) 8-positive and CD4-positive T cells were strongly expressed following hypothermia. Therefore, the present study provided evidence that IL-2 treatment combined with MFH improves the therapeutic effect on lung cancer-bearing mice.
\end{abstract}

\section{Introduction}

Lung cancer is one of the most common malignant tumors in the world. Current therapeutic options remain unsatisfactory for the majority of patients. Surgical resection has been recognized as the most effective method for the treatment of lung cancer; however, it is available only for a small number of patients (1). Therefore, it is crucial to identify a new method to treat lung cancer (2).

Magnetic fluid hyperthermia (MFH) is a thermal therapy using nanotechnology and hyperthermia (3). Dispersions of biocompatible iron oxide nanoparticles in water (magnetic fluids) can be injected into tumors and heated in an externally

Correspondence to: Dr Shenglin Ma, Department of Radiation Oncology, Hangzhou First People's Hospital, Nanjing Medical University, 261 Huansha Road, Hangzhou, Zhejiang 310006, P.R. China E-mail: mashenglin@medmail.com.cn

Key words: magnetic fluid, hyperthermia, interleukin-2, lung cancer applied alternating magnetic field (AMF) by Brownian and Neel relaxation mechanism (4). As the magnetic particles were directly injected into tumors, there was no distribution of magnetic particles in the periphery of normal tissues and the temperature of these normal tissues did not increase significantly. Therefore, the hyperthermia was specifically targeted to the tumors. The efficacy of hyperthermia was demonstrated using MFH in animals with several types of tumor, such as B16 mouse melanoma, T-9 rat glioma, SMMC-7721 mouse hepatocarcinoma and BT-474 mouse breast cancer (5-8). This method was effective in inducing regression of tumors and increasing the life span of the animals. As a result, MFH appears to be a promising method for targeting lung cancer.

Immunotherapy is an accepted therapeutic modality. Interleukin-2 (IL-2) is a significant stimulator of lymphocyte proliferation and enhances cytotoxic $\mathrm{T}$ lymphocytes (CTLs) activity. IL-2 has a wide range of immunological effects, including induction of specific T helper cells, natural killer (NK) cells and lymphokine-activated killer cells (9). Due to these effects, IL-2 is broadly used in cancer therapy to improve cellular immunity and the cytotoxic activity of effector cells (10).

Based on these results, the present study examined the feasibility of combining IL-2 and MFH for lung cancer treatment. To the best of our knowledge, for the first time the combination of a local injection of IL-2 and MFH has been shown to exhibit an evident antitumor effect against lung cancer.

\section{Materials and methods}

Cell line and animal model. Lewis lung cancer cells were purchased from the Institute of Biochemistry and Cell Biology (Shanghai Institute of Biological Sciences, Institute of Materia Medica, Chinese Academy of Medical Sciences, Shanghai, China) and supplemented with $10 \%$ fetal bovine serum.

A total of 60 C57/BL6 mice (male, 8 weeks old) were purchased from the Shanghai Institute of Biological Sciences (Chinese Academy of Medical Sciences). The mice were fed with sterile food and water, housed in sterile cages on sterile bedding, and placed in rooms with a constant temperature and humidity. To prepare the tumor model, the cell density was adjusted to $\sim 1.0 \times 10^{7} / \mathrm{ml}$. To induce tumors, $0.2 \mathrm{ml}$ of Lewis lung cancer cell suspension ( $2 \times 10^{6}$ cells) was injected into the right flank of each mouse through hypodermic inoculation. The noticeable tumors were observed 4 days after inoculation. The size of the tumors 
was measured by vernier calipers every day, and the tumor volume was calculated using the following formula:

$$
\mathrm{V}=1 / 2 \mathrm{ab} \mathrm{b}^{2} \mathrm{~cm}^{3}
$$

Where ' $a$ ' is the major diameter of the tumor and ' $b$ ' is the minor diameter perpendicular to the major diameter (in $\mathrm{cm}$ ).

The treatment started when the major diameter was $\sim 0.8 \pm 0.1 \mathrm{~cm}$.

All the animal experiments were performed according to the principles described in the Guide for the Care and Use of Laboratory Animals as promulgated by the Zhejiang Standing Committee (Zheijiang, China).

Hyperthermia. The mice were randomly selected and anesthetized with $2 \%$ pentobarbital sodium (Beijing Reagent Co., Beijing, China) by intraperitoneal injection $(50 \mathrm{mg} / \mathrm{kg})$. The mice were divided into four groups $(\mathrm{n}=15)$ : Group I (control), II (MFH), III (IL-2) and IV (MFH+IL-2). In groups II and IV, $15 \mathrm{mg}$ of magnetic fluid was slowly injected into the tumors with a 1-ml syringe. Following the magnetic fluid injection $(24 \mathrm{~h}$ later), the tumors of mice in groups II and IV were subjected to AMF for $30 \mathrm{~min}$. MFH was induced using a separated high-frequency induction heating machine (Type SP-04AC 4 KW150 kHz; Shenzhen Power Supply Technology, Guandong, China). Tumor and rectum temperature during AMF irradiation were measured by an optical fiber probe (YF-200). The temperature was maintained at $43^{\circ} \mathrm{C}$ for $30 \mathrm{~min}$ by controlling the strength of the magnetic field. Mice in groups III (IL-2) and IV (MFH+IL-2) were injected with recombinant IL-2 (5x10 4 units; Beijing Four Rings Biological Pharmaceutical Co., Ltd., Beijing, China), after $24 \mathrm{~h}$ of hyperthermia, and IL-2 was injected directly into the nodules. Administration of cytokines was carried out every day for 2 days.

After 14 days, all the mice were sacrificed by neck dislocation. The weight and volume inhibitory rates (IW and IV, respectively) of the tumor were calculated as follows:

IW $=(1-$ experimental group tumor weight/control group tumor weight $) \times 100 \%$; and IV $=(1-$ experimental group tumor volume/control group tumor volume) x $100 \%$.

Pathological observation. The day after hyperthermia, 3 mice were randomly chosen from each group and sacrificed by neck dislocation. The tumors were taken out immediately and dissected. The resected tumors were fixed in $10 \%$ formalin, embedded in paraffin, sectioned and stained with hematoxylin-eosin, which was subsequently followed by histological observation on the tumors.

Preparation of specimens for immunohistochemical (IHC) staining. After $24 \mathrm{~h}$ of hyperthermic treatment, tumors were removed and specimens for IHC staining were prepared. For immunostaining of heat-shock protein 70 (HSP70), cluster of differentiation 4 (CD4) and CD8, the resected tumors were fixed in $10 \%$ formalin solution and embedded in paraffin. Sections (4- $\mu \mathrm{m})$ of paraffin-embedded specimens were deparaffinized in xylene and rehydrated with a series of ethanol washes. Autoclave treatment was performed at $120^{\circ} \mathrm{C}$ for $10 \mathrm{~min}$ in $10 \mathrm{mM}$ sodium citrate buffer ( $\mathrm{pH}$ 6.0) for antigen retrieval. The paraffin-embedded sections were incubated at $37^{\circ} \mathrm{C}$ for

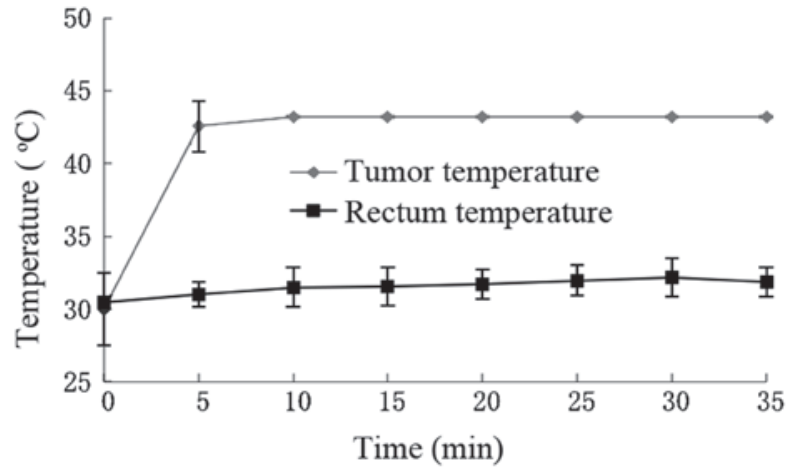

Figure 1. Induction of hyperthermia using an alternating magnetic field $24 \mathrm{~h}$ after magnetic fluid was injected. The tumor temperature was increased to $>43^{\circ} \mathrm{C}$ within $5 \mathrm{~min}$, and was maintained at $\sim 43^{\circ} \mathrm{C}$ by controlling the strength of the alternating magnetic field. The rectum temperature was maintained at $\sim 30^{\circ} \mathrm{C}$.

60 min with mouse monoclonal antibody preparations (MAbs) and with rat MAbs against HSP70, CD4 and CD8 (1:1,000; BD PharMingen, San Jose, CA, USA) antigens, respectively. These sections were subsequently incubated at $37^{\circ} \mathrm{C}$ for 60 min with biotinylated goat anti-mouse immunoglobulin $\mathrm{G}$ (IgG) or biotinylated mouse anti-rat $\operatorname{IgG}$ (Boster Co., Wuhan, China). Specimens were incubated at $37^{\circ} \mathrm{C}$ for 30 min with alkaline phosphatase. Each step was followed by washing with phosphate-buffered saline (PBS). Alkaline phosphatase and peroxidase activities were visualized using the New Fuchsin Substrate System (Shanghai Xin Yu, Biotechnology Co., Ltd., Shanghai, China) and diaminobenzidine tetrahydrochloride solution containing $0.005 \%$ hydrogen peroxide, respectively. All slides were counterstained with hematoxylin. For the negative controls, primary antibodies were replaced with either unrelated monoclonal antibodies or PBS.

Statistical analysis. To evaluate the significance of overall differences in tumor volumes and weights among all the in vivo groups, statistical analysis was performed by analysis of variance. $\mathrm{P}<0.05$ was considered to indicate a statistically significant difference. The tumor volumes and weight data are represented as mean \pm standard error.

\section{Results}

Hyperthermia by means of magnetic fluid in vivo. During hyperthermia, the tumor temperature increased to $>43^{\circ} \mathrm{C}$ in 5 min and fluctuated $\sim 43^{\circ} \mathrm{C}$ with controlled strength of AMF. The rectum temperature remained $\sim 30^{\circ} \mathrm{C}$ (Fig. 1).

Histological analysis. Following hyperthermia, in groups II and IV, the surface of the majority of tumors scabbed and local necrosis occurred after 2-3 days. A large number of apoptotic tumor cells were discovered with the characteristics of karyopyknosis and condensation. In addition, there was coagulation necrosis in certain areas. The acidophilia increased and normal cell structure disappeared. Hemorrhagic lesions were detected in certain apoptotic and necrotic areas. There were numerous red blood cells around the necrotic tumor cells and angiorrhexis had occurred. The magnetic fluid was distributed between the apoptotic and necrotic areas (Fig. 2). 
Table I. Volume and mass inhibitory rates of Lewis lung cancer in nude mice following treatment.

\begin{tabular}{llccc}
\hline Groups & $\begin{array}{c}\text { Tumor volume, } \\
\text { mean } \mathrm{mm}^{3} \pm \mathrm{SE}\end{array}$ & $\begin{array}{c}\text { Volume } \\
\text { inhibitory rate, } \%\end{array}$ & $\begin{array}{c}\text { Tumor mass, } \\
\text { mean g } \pm \text { SE }\end{array}$ & $\begin{array}{c}\text { Mass inhibitory } \\
\text { rate, } \%\end{array}$ \\
\hline Control & $4,459.8 \pm 2,044.1$ & - & $8.31 \pm 4.11$ & $45.8^{\mathrm{a}}$ \\
MFH & $2,775.1 \pm 90.4$ & $37.8^{\mathrm{a}}$ & $4.51 \pm 0.25$ & $1.13 \pm 2.40$ \\
IL-2 & $3,875.8 \pm 1,284.1$ & $13.1^{\mathrm{b}}$ & $2.65 \pm 1.21$ & $68.1^{\mathrm{a}}$ \\
MFH+IL-2 & $1,277.8 \pm 645.9$ & $71.4^{\mathrm{a}}$ & & \\
\hline
\end{tabular}

${ }^{\mathrm{a}} \mathrm{P}<0.01$ and ${ }^{\mathrm{b}} \mathrm{P}>0.05$ vs. control group. SE, standard error; MFH, magnetic fluid hyperthermia; IL-2, interleukin-2.

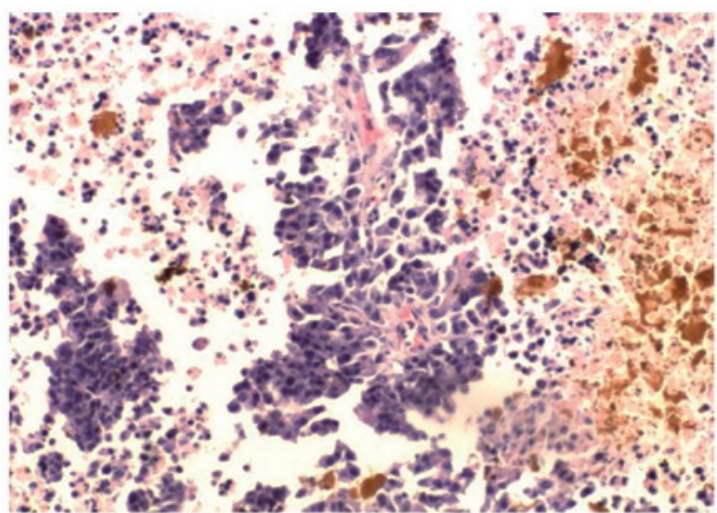

Figure 2. Magnetic fluid is distributed between the apoptotic and necrotic areas, with a significant number of apoptotic and coagulation necrosis tumor cells in certain areas following hyperthermia (magnification, $\mathrm{x} 400$ ).

In groups I and III, the sizes of tumors were increased with time and were regular with a smooth surface. The nucleoli were stained and karyokinesis was occasionally detected. There were a few ischemic necrotic tissues observed in the center.

IHC of Lewis lung cancer following MFH. Expression of HSP70, CD4-positive and CD8-positive T cells in Lewis lung cancer was investigated and the results are shown in Fig. 3. Without hyperthermia, staining for HSP70, CD4 and CD8 had negative outcomes (Fig. 3A). However, following hyperthermia, HSP70, CD4 and CD8 were strongly expressed in the viable cells around the necrotic area, as shown in Fig. 3B-D.

Antitumor effects of hyperthermia. In groups I and III, the tumors grew progressively. In groups II and IV, the tumors grew slowly. On day 14 after treatment, the mass and volume inhibitory ratios of groups III and IV were IM=45.8 and $68.1 \%$, and IV=37.8 and $71.4 \%$, respectively. Therefore, the ratio of group IV was much higher than that of the other groups (Table I).

\section{Discussion}

MFH represents an innovative technique to heat deep-seated tumors, such as lung cancer. It may have several merits over conventional techniques employed for regional hyperthermia, including radiofrequency, microwave and ultrasound methods, which are often limited by their inability to selectively target the tumor tissue. The method of MFH is able to heat the specific tumor. Furthermore, the temperature during hyperthermia can be controlled by AMF, which allows the operator to induce the tumor cell apoptosis and necrosis without damaging the surrounding normal tissue.

Hyperthermia induces the expression of HSPs (11), such as HSP70, HSP90 and glucose-regulated protein 96. HSPs have a chaperone function for tumor antigens. The mechanism of antitumor immunity induced by MFH is possibly a proposed mechanism regarding antigen presentation by HSP70 expression during hyperthermia (12). One such mechanism is heat-induced enhancement of antigenic peptide presentation through major histocompatibility complex class I antigens of tumor cells, and therefore, enhanced tumor immunogenicity and subsequently augmented T-cell cross-priming (13). These results have suggested that HSP70 is an important modulator of tumor-cell immunogenicity during hyperthermia and CTLs are the effector cells.
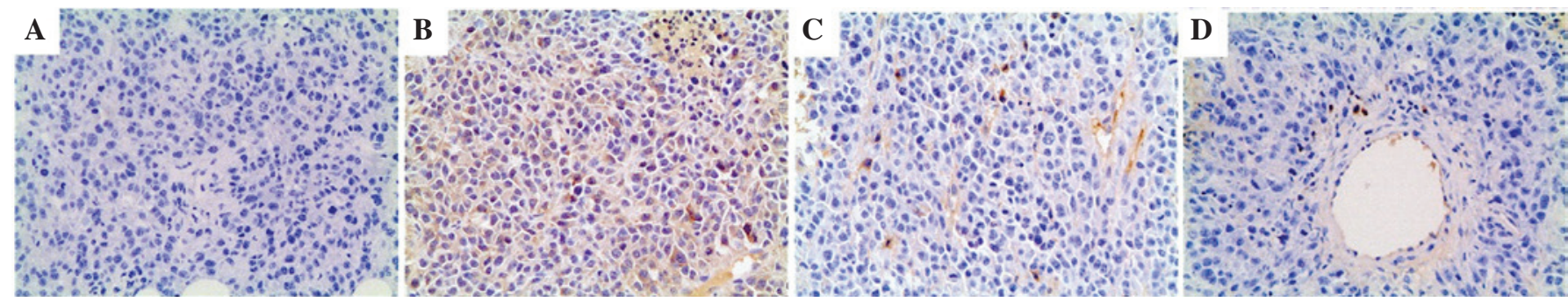

Figure 3. Expression of HSP70, CD4-positive and CD8-positive T cells in Lewis lung cancer. (A) Without hyperthermia, staining for HSP70, CD4 and CD8 was negative. (B-D) However, following hyperthermia, HSP70, CD4, and CD8 were strongly expressed in the viable cells around the necrotic area (magnification, $\mathrm{x} 200)$. HSP, heat-shock protein; $\mathrm{CD}$, cluster of differentiation. 
The most noteworthy finding of the present study was that MFH combined with IL-2 improves the therapeutic effect on Lewis lung cancer in mice. IL-2 is a cytokine from the cytokine-receptor chain family with numerous functions, including stimulating the proliferation of $\mathrm{T}$ cells, inducing the production of NK cells and CTL generation, and facilitating the synthesis of immunoglobulins produced by B cells $(14,15)$. IL-2 has been used in antigen-presenting cell-based tumor vaccines (16). This has already been approved for clinical use in patients with metastatic renal cell carcinoma and malignant melanoma $(17,18)$. The study demonstrated that there was no substantial expression of CD4 or CD8 in the tumors injected with IL-2 and essentially the growth of the tumors was not inhibited, which suggests that IL-2 alone has a poor antitumor effect on lung cancer. This may be associated with the correlation between the implication of IL-2 in the immunological regulation and the self-regulation of $\mathrm{T}$ lymphocytes, although the exact mechanism remains to be elucidated. In contrast to group III, the growth of the tumors in groups II (MFH) and IV (IL-2+MFH) was significantly inhibited, with the inhibition being more robust in group IV, suggesting that IL-2 could enhance the antitumor effect of MFH against lung cancer.

In conclusion, the present study has provided evidence that IL-2 treatment combined with MFH improved the therapeutic effect on lung cancer.

\section{Acknowledgements}

The present study was supported by grsants from the Medicine and Health Foundation of Zhejiang Province (no. 2009A163), the Science and Technology Development Foundation of Hangzhou (no. 20080333B02), and Medicine and Health Foundation of Hangzhou (no. 2012Z002).

\section{References}

1. Amorín Kajatt E: Lung cancer: A review of current knowledge, diagnostic methods and therapeutic perspectives. Rev Peru Med Exp Salud Publica 30: 85-92, 2013 (In Spanish).

2. Zhang Y and Li X: Clinical application and advances in radiofrequency ablation of lung neoplasms. Zhongguo Fei Ai Za Zhi 13: 1064-1069, 2010 (In Chinese).

3. Li XH, Rong PF, Jin HK, Wang W and Tang JT: Magnetic fluid hyperthermia induced by radiofrequency capacitive field for the treatment of transplanted subcutaneous tumors in rats. Exp Ther Med 3: 279-284, 2012.
4. Thiesen B and Jordan A: Clinical applications of magnetic nanoparticles for hyperthermia. Int J Hyperthermia 24: 467-474, 2008.

5. Ito A, Matsuoka F, Honda $\mathrm{H}$ and Kobayashi T: Antitumor effects of combined therapy of recombinant heat shock protein 70 and hyperthermia using magnetic nanoparticles in an experimental subcutaneousmurine melanoma. Cancer Immunol Immunother 53: 26-32, 2004

6. Yanase M, Shinkai M, Honda H, Wakabayashi T, Yoshida J and Kobayashi T: Antitumor immunity induction by intracellular hyperthermia using magnetite cationic liposomes. Jpn J Cancer Res 89: 775-782, 1998.

7. Wang ZY, Song J and Zhang DS: Nanosized $\mathrm{As}_{2} \mathrm{O}_{3} / \mathrm{Fe}_{2} \mathrm{O}_{3}$ complexes combined with magnetic fluid hyperthermia selectively target liver cancer cells. World J Gastroenterol 15: 2995-3002, 2009.

8. Kikumori T, Kobayashi T, Sawaki M and Imai T: Anti-cancer effect of hyperthermia on breast cancer by magnetite nanoparticle-loaded anti-HER2 immunoliposomes. Breast Cancer Res Treat 113: 435-441, 2009.

9. Liao W, Lin JX and Leonard WJ: Interleukin-2 at the crossroads of effector responses, tolerance and immunotherapy. Immunity 38 : 13-25, 2013.

10. Chou SH, Shetty AV, Geng Y, Xu L, Munirathinam G, Pipathsouk A, Tan I, Morris T, Wang B, Chen A and Zheng G: Palmitate-derivatized human IL-2: A potential anticancer immunotherapeutic of low systemic toxicity. Cancer Immunol Immunother 62: 597-603, 2013.

11. Wang L, Dong J, Ouyang W, Wang X and Tang J: Anticancer effect and feasibility study of hyperthermia treatment of pancreatic cancer using magnetic nanoparticles. Oncol Rep 27: 719-726, 2012.

12. Tanaka K, Ito A, Kobayashi T, Kawamura T, Shimada S, Matsumoto K, Saida T and Honda H: Intratumoral injection of immature dendritic cells enhances antitumor effect of hyperthermia using magnetic nanoparticles. Int J Cancer 116: 624-633, 2005.

13. Torigoe T, Tamura $\mathrm{Y}$ and Sato N: Heat shock proteins and immunity: Application of hyperthermia for immunomodulation. Int J Hyperthermia 25: 610-616, 2009.

14. Beyer M: Interleukin-2 treatment of tumor patients can expand regulatory T cells. Oncoimmunology 1: 1181-1182, 2012.

15. Quintarelli C, Savoldo B and Dotti G: Gene therapy to improve function of $\mathrm{T}$ cells for adoptive immunotherapy. Methods Mol Biol 651: 119-130, 2010.

16. Yang JY, Li X, Gao L, Teng ZH and Liu WC: Co-transfection of dendritic cells with AFP and IL-2 genes enhances the induction of tumor antigen-specific antitumor immunity. Exp Ther Med 4: 655-660, 2012.

17. Rosenberg SA: Raising the bar: The curative potential of human cancer immunotherapy. Sci Transl Med 4: 127ps8, 2012.

18. Ellebaek E, Engell-Noerregaard L, Iversen TZ, Froesig TM, Munir S, Hadrup SR, Andersen MH and Svane IM: Metastatic melanoma patients treated with dendritic cell vaccination, Interleukin-2 and metronomic cyclophosphamide: Results from a phase II trial. Cancer Immunol Immunother 61: 1791-1804, 2012. 